\title{
Representações Sociais de saúde construídas por idosos do oeste paraense
}

\section{Sodal representationson hedth built by theedderly of nestem Parastate, Brazil}

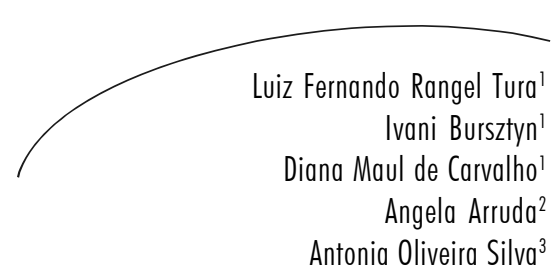

Resumo

Introdução: Objetivando identificar questões relevantes para o monitoramento de agravos à saúde de idosos decorrentes da pavimentação da rodovia CuiabáSantarém (BR-163), procurou-se apreender os sentidos de saúde construídos por este grupo, fundamentando-se na teoria das representações sociais. Metodologia: Investigou-se amostra aleatória de 89 pessoas com mais de 60 anos de dois municípios na área de influência dessa rodovia. Utilizou-se um teste de evocação livre de palavras com o termo "saúde" e um questionário com perguntas abertas e fechadas para caracterizar conhecimentos, crenças, atitudes e práticas. Resultados: Ficou evidenciada a centralidade de "médicos", o principal organizador dessa representação, e o sistema periférico constituído pelos elementos "hospital", "profissionais”, “prevenção”, “disposição” e "dinheiro”. Conclusões: Os resultados indicam que a carência de infraestrutura assistencial influencia o processo de construção de sentidos de saúde. Isto adquire maior relevância diante da perspectiva de pavimentação da rodovia BR-163, que poderá trazer incremento dos agravos à saúde.

\section{Abstract}

Introduction: To contribute to the development of health care for the elderly, and identify aspects of health perception useful for monitoring strategies of health hazards linked to the paving of Cuiabá-Santarém Highway (BR-163), social representations of health by the elderly were studied through theory of social

\footnotetext{
Laboratório de História, Saúde e Sociedade, Departamento de Medicina Preventiva, Faculdade de Medicina. Universidade Federal do Rio de Janeiro. Rio de Janeiro, RJ, Brasil.

2 Departamento de Psicologia Social, Instituto de Psicologia. Universidade Federal do Rio de Janeiro. Rio de Janeiro, RJ, Brasil.

3 Programa de Pós-Graduação em Enfermagem. Universidade Federal da Paraíba. João Pessoa, PB, Brasil.

Financiamento: CNPq - Proc. 402867/05-0

Aprovado pelo Comitê de Ética da Pesquisa da FIOCRUZ (Proc. 0022.0.011.011-06)
}

Palavras-chaves: Saúde. Idoso. Saúde do idoso. Medicina Preventiva. Representações sociais. 
representations. Methodology: A random sample of 89 people aged 60 or over was investigated. Association test with the word "health" and a questionnaire were used. Results: Analysis showed the element "physicians" composing the central system and "hospital”, "professionals", "prevention”, "disposition” and "money”, the peripheral system. Conclusions: The lack of health assistance influences the build up of health meanings. Results are even more relevant as the paving of BR163 may bring on new health hazards.
Key words: Health. Aged. Health of the Elderly. Preventive Medicine. Social representations.
INTRODUÇÃO

Em países como o Brasil, de dimensões continentais e grandes contrastes socioeconômicos, observa-se que a preocupação com o envelhecimento se concentra nos grandes centros urbanos. Em áreas mais distantes, as necessidades dos idosos podem estar sendo negligenciadas, em vista de prioridades emergenciais da população mais jovem. Neste estudo, tem-se como objetivo contribuir para o aperfeiçoamento de medidas de atenção à saúde dos idosos na área de influência da rodovia CuiabáSantarém (BR-163), a partir da apreensão das representações sociais de saúde construídas por esse grupo.

Tem sido uma constatação recorrente que os serviços de saúde, públicos e privados, ao implantarem programas dirigidos à prevenção de "problemas do envelhecimento" - principalmente doenças crônicas - assumem a perspectiva de que os indivíduos têm condutas saudáveis na medida de sua avaliação pessoal de riscos e benefícios para a saúde, apesar de tal comportamento não ser observado em muitos casos quando se trata de prevenção primária. ${ }^{1,2}$

As ações educativas propostas nem sempre contemplam a complexidade do processo de envelhecimento, desconsiderando as várias dimensões envolvidas na apropriação de conhecimentos e habilidades necessárias ao desenvolvimento de condutas saudáveis. ${ }^{3}$ De fato, as experiências exitosas, como de North Karelia, foram obtidas através de mudanças em toda a sociedade e não somente no nível individual. ${ }^{4}$ Assim, vários outros fatores - incluindo o tempo, os espaços de convivência e a interdependência entre diferentes dimensões socioculturais - estão envolvidos quando se propõe analisar o problema do envelhecimento populacional.

Em nosso meio, os dados epidemiológicos indicam que a demanda por consultas médicas está relacionada sobretudo a cardiopatias, hipertensão arterial, diabetes e afecções osteoarticulares. Além disso, esse grupo está sujeito a outras situações ligadas à condição de aposentadoria, pouco convívio social e familiar, além da incapacidade de se cuidar com autonomia e as apreensões relativas à proximidade da morte. ${ }^{5}$ Há ainda que se considerar que a conjuntura de iniquidade socioeconômica existente no país desenha formas desiguais de acesso aos serviços de saúde e a outros bens e recursos sociais. Observa-se, com isso, entraves à integralidade da

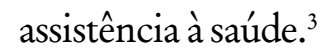

Pesquisa realizada em Juiz de Fora (MG) identificou que, para os idosos, a imagem do idoso saudável está relacionada com ser capaz de manter a saúde física e emocional, poder contar com o apoio familiar e do grupo social, dedicarse a atividades que dão prazer, ter hábitos de vida saudáveis, manter a fé e desempenhar as atividades do cotidiano. Enquanto isso, seus cuidadores enfatizam a autonomia e independência. ${ }^{6}$

O crescimento do contingente de idosos na população reforça, para esse grupo, o que foi assinalado por Pellegrini-Filho ${ }^{7}$, quando destaca a importância de se assumir, em relação à saúde, a necessidade de encontrar alternativas metodológicas que permitam desenvolver pesquisas baseadas em marcos explicativos mais amplos do que os proporcionados pelo uso dos indicadores tradicionais.

Nesta linha de análise, o que está em curso é a necessidade de se buscar uma nova perspectiva na 
abordagem dos fenômenos da saúde, que contemple as dimensões subjetivas na relação saúde e doença, introjetadas nos indivíduos através dos processos da convivência sociocultural. ${ }^{8} \mathrm{Ou}$ seja, estão em curso situações complexas, que articulam dimensões sociais com aspectos constituídos a partir da mobilização de valores, crenças, formas de entender a vida, a saúde, as relações com o corpo e os outros; e que afetam fortemente os modos do indivíduo e dos grupos se relacionarem com as diversas circunstâncias do existir, entre elas a questão do envelhecimento.

Moscovici ${ }^{8}$ assinala que a observação do crescente consumo na área da medicina, o aumento da difusão de técnicas e teorias médicas e as mudanças na relação médico-paciente possibilitaram transformações de imagens e teorias antigas existentes no campo da saúde. E Arruda ${ }^{9}$ destaca as ressignificações nas concepções sobre saúde e doença, que essas transformações propiciaram. $\mathrm{O}$ escopo de análise desses objetos se ampliou, incluindo a contribuição das ciências sociais para o entendimento de aspectos culturais, étnicos, religiosos etc., que constroem as visões pessoais sobre a saúde e a doença e definem condutas diante das novidades que muitas doenças revelam em suas etiologias e formas de apresentação.

Cuidar do corpo e da saúde é entendido, a partir do século XX, como tarefa ou dever do cidadão. A questão sanitária ganhou outras dimensões, expandiu-se e, assim, os objetos de pesquisa nesse campo adquiriram novos sentidos e significados, e seu estudo passou a exigir "pelo menos duas direções aparentemente opostas: a do contexto e a do sujeito para chegar a um destino:

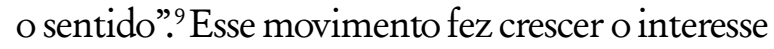
pela teoria das representações sociais, percebida como aporte importante no estudo das direções e sentidos das mudanças. Arruda ${ }^{9}$ distingue a capacidade da teoria das representações sociais, em sua interface com várias áreas do conhecimento, de captar o movimento subjetivo e compreender/entender a realidade social, sem se desconectar de seus vínculos com as formas de organização social, as demandas das diferentes culturas e as características de um tempo e lugar.
As representações sociais constituem um tipo de conhecimento de elaboração coletiva, socialmente partilhado, "concorrendo para a construção de uma realidade comum a um conjunto social". ${ }^{10}$ De fato, em suas condutas e práticas cotidianas, os profissionais de saúde e a população atendida trocam informações e noções, permeadas por ideias, símbolos, valores, normas, estereótipos que se enraízam em suas histórias e vivências e, nesse processo, o objeto se torna familiar e se naturaliza.

Rezende, ${ }^{11}$ visando a identificar as demandas e necessidades de atenção à saúde, por meio dos sentidos que idosos atribuem à consulta médica, assinala que o grupo investigado representava a consulta médica em duas perspectivas: uma técnica, em que o idoso espera encontrar na consulta a competência médica e a orientação para o tratamento; e uma dialógica, em que há expectativa de encontrar um médico amigo e solidário, disposto a estabelecer com o cliente uma conversa pautada pela troca de informações. Esses resultados ressaltam a importância dos serviços de saúde, não apenas como espaço de assistência a doenças, mas também como possibilidade de apoio afetivo e na construção de redes sociais.

Situando o indivíduo em seu contexto sóciohistórico, animado de suas práticas e experiências cotidianas, a teoria das representações sociais permite reunir dados que integram redes de significados e trazem subsídios para avaliar a forma complexa de apropriação e reconstrução de sentidos atribuídos aos objetos - sentidos estes que têm implicações diretas sobre as condutas e os processos comunicacionais. ${ }^{12}$

\section{CONTEXTO DO ESTUDO}

Os dados aqui apresentados visam a caracterizar as condições de vida e saúde da população estudada. Os municípios estudados Santarém e Belterra - situam-se no oeste do estado do Pará, na área de influência direta da rodovia Cuiabá-Santarém (BR-163), que está em processo de pavimentação. As transformações esperadas nos contextos ambiental, econômico e social geram 
expectativas quanto a mudanças demográficas, culturais, sanitárias e outras.

Santarém é o segundo maior município do estado, com uma população estimada em 276.663 habitantes para o ano de 2009, enquanto para Belterra, a estimativa para o mesmo ano foi de 12.672 habitantes. Em Santarém, a população abaixo de 20 anos corresponde a $41,7 \%$ do total e os idosos (acima de 60 anos) perfazem $8 \%$. Em Belterra esses valores são, respectivamente, $44,8 \%$ e $7,7 \%{ }^{13}$

Com maior grau de urbanização Santarém apresentava, no ano $2000,59,5 \%$ dos moradores servidos pela rede geral de abastecimento de água, enquanto a situação das instalações sanitárias se mostrava mais precária, com $61 \%$ dos moradores utilizando fossa rudimentar. Em Belterra esses valores eram, respectivamente, $27,7 \%$ e $93,8 \% .^{13}$

De acordo com dados de 2006, em Santarém, entre as principais causas de morte destacam-se as doenças do aparelho cardiovascular, do aparelho respiratório, causas externas e neoplasias. As mortes por doenças cardiovasculares correspondem a $30 \%$ do total das mortes e $44,8 \%$ das mortes entre os de 60 anos e mais. Em Belterra, para o mesmo período, entre as principais causas de morte destacam-se as do aparelho cardiovascular, aparelho respiratório, neoplasias e causas externas. As mortes por doenças cardiovasculares correspondem a $27 \%$ do total e a $40 \%$ entre os de 60 anos e mais. O grupo de causas mal definidas correspondeu a $42 \%$ em Belterra e $19,8 \%$ em Santarém. ${ }^{13}$

A rede de serviços de assistência à saúde em Belterra é constituída por três unidades básicas, três postos de saúde e uma unidade mista da antiga Fundação SESP. Com base no Sistema de Informação Hospitalar (SIH), para o ano de 2007, a taxa de internação da população residente em Belterra foi de 3,1/100 habitantes; a oferta de leitos era de 1,0/1.000 habitantes; e o município contava com 0,3 médicos $/ 1.000$ habitantes. O município de Santarém dispõe de uma rede complexa, com um total de 149 estabelecimentos, sendo 95 públicos, um filantrópico e 53 privados. A taxa de internação hospitalar da população residente em Santarém, para o mesmo ano, foi de 5,9/100 habitantes, e a oferta local de leitos era de 1,7/1.000 habitantes. O município contava com 1,5 médicos/ 1.000 habitantes. As principais causas de internação em Belterra foram, para 2007, "doenças infecciosas e parasitárias" $(18,6 \%)$, "gravidez, parto e puerpério" (18,5\%) e "doenças do aparelho genitourinário" (16,1\%). Em Santarém, as principais causas de internação foram "gravidez, parto e puerpério" (46\%), "doenças do aparelho respiratório" (19,8\%) e "doenças infecciosas e parasitárias" (10,2\%). Para o grupo de 60 anos e mais, a principal causa de internação são as doenças cardiovasculares em ambos os municípios. ${ }^{13}$

Santarém é o município-polo da região, contando com maior oferta de serviços não apenas em termos de volume, mas também pelo nível de complexidade ofertada (exames de mamografia, tomografia computadorizada, ultrassonografia, ressonância magnética e outros). No entanto, apesar das evidentes diferenças entre os dois municípios, é patente a carência de capacidade de atenção refletida na baixa oferta de leitos e baixa taxa de internação hospitalar, mesmo quando se comparam as médias nacionais. ${ }^{14}$

\section{METODOLOGIA}

O grupo estudado foi constituído por 89 idosos - pessoas com 60 anos e mais -, integrantes de um conjunto de 695 sujeitos de ambos os sexos, residentes nos dois municípios do oeste paraenses. Nos centros urbanos, os sujeitos foram selecionados a partir de amostra aleatória obtida por sorteio de famílias cadastradas no Programa de Saúde da Família em Santarém e Belterra (1.345 e 1.059 famílias), correspondendo a 302 e 201 famílias, respectivamente. $\mathrm{Na}$ área rural de Santarém, São José, foram incluídas todas as famílias (320) e entrevistados os sujeitos maiores de 16 anos que estavam em casa e concordaram em participar da pesquisa no dia do trabalho de campo, correspondendo a 192 famílias.

A investigação teve como fundamento teóricometodológico a abordagem estrutural das 
representações sociais ${ }^{15}$, utilizando-se um teste livre de associação com o termo indutor "saúde" e um questionário com respostas abertas e fechadas visando a categorizar os sujeitos em suas características sociodemográficas e apreender normas, valores, atitudes e práticas em relação à saúde. O material obtido foi analisado de acordo com suas respectivas especificidades. $\mathrm{O}$ corpus oriundo das evocações foi organizado segundo uma estratégia que permite articular as dimensões individual e coletiva através da verificação das frequências - dimensão coletiva - e das ordens médias de evocação - dimensão individual, identificando-se o conteúdo da representação social. ${ }^{16,17}$

Seguindo-se essa orientação, foram calculadas as médias das frequências e ordens médias de evocação para, em seguida, lançar esses parâmetros em um gráfico de dispersão onde o cruzamento das respectivas linhas o dividirá em quadrantes. ${ }^{13,19}$ Nessa perspectiva, os elementos que são mais prontamente evocados e com maiores frequências localizam-se no quadrante superior esquerdo, possivelmente constituintes do sistema central da representação social. No quadrante inferior direito, estarão os elementos de características opostas e participantes do sistema periférico. Nos outros dois quadrantes, situam-se os elementos com propriedades mistas, compondo o que Flament ${ }^{20}$ denominou de periferia próxima, uma vez que guardam uma relação de proximidade com o sistema central.

Para se analisar como os elementos componentes dessa representação se organizam, foi utilizada a análise de similitude, que permite a apreciação do valor simbólico desses elementos, ao identificar relações significativas entre os conjuntos formados por eles. ${ }^{21,22}$ As repostas abertas tiveram seus conteúdos categorizados segundo técnica preconizada por Bardin, ${ }^{23} \mathrm{e}$ as fechadas, por análise de proporções.

O projeto foi avaliado e aprovado pelo Comitê de Ética da Pesquisa da Fiocruz, e no seu desenvolvimento procurou-se atender aos preceitos vigentes de pesquisa com seres humanos.

\section{RESULTADOS}

$\mathrm{Na}$ amostra estudada, observaram-se $43,8 \%$ de mulheres e 56,2\% de homens, com a idade variando entre 92 e 60 anos, estando a moda na faixa de 6064 anos $(43,2 \%)$. Na tabela 1 , pode-se observar que a amostra se constituiu por um grupo de idosos majoritariamente situados entre os 60 e 70 anos.

Tabela 1 - Distribuição dos sujeitos segundo a faixa etária. Santarém e Belterra, PA, 2009.

\begin{tabular}{ccc}
\hline Faixa Etária & $\mathrm{f}$ & $\%$ \\
\hline $60-64$ & 38 & 43,2 \\
$65-69$ & 25 & 28,4 \\
$70-74$ & 10 & 11,4 \\
$75-79$ & 10 & 11,4 \\
$80-84$ & 1 & 1,1 \\
$85-89$ & 3 & 3,4 \\
$90-94$ & 1 & 1,1 \\
Total & 89 & 100,0 \\
\hline
\end{tabular}

Quanto às condições de moradia, 64,1\% apresentavam boas condições de conservação, dividindo-se em $47,2 \%$ de construções de alvenaria e $42,7 \%$ de madeira - material muito utilizado em construções tradicionais na região. Em $65,2 \%$ das residências visitadas, o abastecimento de água vinha da rede pública; entretanto, $15,7 \%$ só contavam com poço artesanal como fonte de água para atendimento de suas necessidades. Quanto aos equipamentos domésticos, as famílias de modo geral eram bem servidas, encontrando-se fogão a gás em $80 \%$ das 
casas, geladeira em $87,6 \%$ e televisão em $88,8 \%$. Esses resultados guardam semelhança com os encontrados por Barreto et al., ${ }^{24}$ quando investigaram o perfil sócio-epidemiológico e demográfico de idosas pernambucanas.

Dentre os principais problemas de saúde que informaram apresentar, os cinco primeiros $(74,7 \%)$ referem-se a: "hipertensão arterial" $(23,6 \%)$, "diabetes" (17,1\%), "gripe" (13,8\%), "reumatismo" $(11,7 \%)$ e "diarreia” (8,5\%). Quanto à hipertensão arterial e reumatismo, as proporções referidas foram menores que as encontradas em estudos que investigaram grupos semelhantes ${ }^{25,26,27}$, e em relação ao diabetes, o resultado encontrado é semelhante aos descritos por Lebrão e Laurent $\mathrm{i}^{25}$ para a cidade de São Paulo.

Para analisar o material evocado, inicialmente foi realizada uma "leitura flutuante" para homogeneizar o corpus, visando a superar formas simultâneas de singular/plural, masculino/ feminino, sinônimos etc. ${ }^{22}$ Em seguida, procedeuse ao cálculo das médias das frequências e das ordens médias de evocações e à construção do gráfico de dispersão com os parâmetros obtidos.

Foram constatadas 319 evocações - 3,6 evocações/sujeito - de 96 palavras diferentes, com a média de frequência igual a 18 e média das ordens médias de evocação (OME) igual a 2,5. Esses parâmetros permitiram identificar no quadrante superior esquerdo os elementos "médicos" e "hospital", provavelmente pertencentes ao sistema central desta representação; no quadrante inferior direito, foram encontrados os elementos "profissionais", "prevenção", "disposição" e "dinheiro", compondo o sistema periférico desta estrutura; nos demais quadrantes, encontram-se "remédio", "atendimento", "posto de saúde", "bemestar”, "alegria”, "vida”, "alimentação”, “doença”, “importante” e “descaso” (quadro 1).

Quadro 1 - Análise das evocações.

\begin{tabular}{|c|c|c|c|c|c|c|}
\hline \multirow[t]{2}{*}{$\mathbf{F}_{\mathbf{M}}$} & \multicolumn{3}{|c|}{$\mathrm{OME}<2,5$} & \multicolumn{3}{|c|}{$\mathrm{OME}>=2,5$} \\
\hline & ELEMENTOS & $\mathrm{F}$ & ome & ELEMENTOS & $\mathrm{F}$ & ome \\
\hline \multirow{2}{*}{$\begin{array}{l}>= \\
18\end{array}$} & Médicos & 30 & 2,400 & \multirow{2}{*}{$\begin{array}{l}\text { Remédio } \\
\text { Atendimento }\end{array}$} & 26 & 2,615 \\
\hline & Hospital & 20 & 2,350 & & 24 & 2,625 \\
\hline \multirow{7}{*}{$\begin{array}{l}< \\
18\end{array}$} & Posto de Saúde & 16 & 2,063 & \multirow{7}{*}{$\begin{array}{l}\text { Profissionais } \\
\text { Prevenção } \\
\text { Disposição } \\
\text { Dinheiro }\end{array}$} & 10 & 2,700 \\
\hline & Bem-estar & 12 & 2,417 & & 6 & 3,000 \\
\hline & Nild & 11 & 2,273 & & 6 & 3,333 \\
\hline & vida & 9 & 1,778 & & & \\
\hline & $\begin{array}{l}\text { Alımentação } \\
\text { Doença }\end{array}$ & 9 & 2,444 & & & \\
\hline & Importante & 7 & 2,429 & & & \\
\hline & Descaso & 5 & 1,200 & & & \\
\hline
\end{tabular}

Os elementos que compõem essa representação remetem a questões muito concretas que são vividas pelos sujeitos: a falta de infraestrutura assistencial. Tal conjuntura se refletiu na avaliação que esses sujeitos efetuaram do atendimento que lhes era prestado pelo SUS nos respectivos municípios: $37,3 \%$ os registraram como muito bom/ bom; 39,8\% regular; e 22,9\% péssimo/ ruim. Esses resultados diferem dos constatados por Veras ${ }^{28}$, ao analisar os dados da PNAD/2003 comparados com os da PNAD/1998, observando uma melhor avaliação em 2003 e assinalando que $83,9 \%$ dos idosos consideraram como muito bom/bom o atendimento do SUS naquele ano. 
Já em 1998, 81,2\% dos idosos consideravam muito bom/bom o atendimento. A avaliação dos serviços de saúde feita pelos idosos deste estudo sugere que a realidade local, norte brasileiro, ainda difere da nacional, mesmo se considerando o tempo decorrido entre os levantamentos das PNADs e esta investigação.

Quando consultados sobre as providências necessárias para melhorar a saúde de sua comunidade, os sujeitos assinalaram "providenciar mais médicos para atendimento" (46,9\%), "aumentar o número de profissionais qualificados" (17,3\%), "construir mais postos de saúde" (12,4\%), "manter e aumentar a oferta de medicamentos" (9,8\%) e "providenciar bom atendimento nas unidades de saúde" $(7,4 \%)$, correspondendo estes conteúdos a $93,8 \%$ do corpus categorizado.

$\mathrm{Na}$ composição do sistema periférico, os elementos "profissionais" e "dinheiro" podem estar espelhando a tensão existente diante das necessidades para tratamento de estados patológicos que apresentem necessidade de consultas médicas, profissionais preparados para $\mathrm{o}$ atendimento em uma perspectiva dialógica como descrita por Rezende ${ }^{11}$, bem como de recursos financeiros para aquisição de medicamentos nem sempre disponíveis nas unidades de saúde.

$\mathrm{Na}$ periferia próxima, encontram-se alguns elementos que apontam para outros sentidos de saúde - "bem-estar", "alegria", "vida", "alimentação" -, contrastando com os elementos centrais, ao mesmo tempo em que se mantém uma relação coerente com os elementos "prevenção" e "disposição" situados no sistema periférico. Essa composição está provavelmente sinalizando que os significados de saúde englobam outras dimensões, além daquelas diretamente relacionadas com o processo de prestação de serviços assistenciais. Aspectos afetivos e relacionais podem, portanto, participar, da visão da saúde como parte incontornável que são da vida das pessoas, que concorrem para o bem-estar de forma geral. Ao mesmo tempo, diante da indagação sobre os sentidos da saúde, sobressai o lugar ocupado pelos aspectos materiais da assistência. Isto alerta para a possibilidade de, diante de pesquisadores vistos como oriundos da sede do saber legítimo, os entrevistados desejarem expressar suas necessidades, na esperança de serem ouvidos por um interlocutor que identificam como possível transmissor desta demanda. ${ }^{29}$

Estas dimensões mais amplas da saúde também foram encontradas nos conteúdos das respostas, quando estas apontaram alguns fatores que interferem positivamente na saúde pessoal: boa alimentação (36,1\%), medidas de higiene (13,9\%), exercícios físicos diários $(12,1 \%)$, bom atendimento médico $(8,2 \%)$ e saneamento $(6,4 \%)$. Ou então, ao relatarem as práticas que realizavam para manter a própria saúde. Nesse caso, as cinco categorias mais frequentes, que corresponderam a $83,2 \%$ do total do corpus, foram: mantendo uma alimentação sadia $(40,8 \%)$, usando os remédios prescritos pelos médicos $(14,4 \%)$, realizando exercícios físicos e caminhadas (12,8\%), adotando medidas de higiene pessoal e doméstica $(8,8 \%)$ e procurando trabalhar $(6,4 \%)$.

É também interessante observar o que foi informado como as cinco principais fontes de aprendizado dos fatores e práticas relatadas: experiência pessoal $(25,6 \%)$, médicos $(20,5 \%)$, pais $(13,2 \%)$, televisão $(8,4 \%)$ e escola $(6,0 \%)$, novamente apontando, para os idosos, o lugar do fator relacional no manejo da saúde.

A observação da árvore máxima de similitude mostra que o elemento "médicos" tem seu valor simbólico confirmado exibindo seu poder de organização, uma vez que em torno dele se conectam cinco elementos - "hospital", "posto de saúde", "remédio", "profissionais" e "dinheiro" que se encontram em diferentes posições na estrutura identificada (figura 1).

A leitura cognitiva deste grafo, como recomendado por Pereira ${ }^{22}$, explicita ainda mais a avaliação que fizeram os sujeitos acerca da importância de assegurar acesso a uma rede de serviços que atenda que atenda às suas necessidades. Aponta também outros sentidos como, por exemplo, quando articula "prevenção" 
com "saneamento", “alimentação" e "atendimento", ou "vida" com "disposição", "bem-estar" e "alegria”. São significados que refletem a saúde fora do sistema assistencial, posicionados no campo da promoção da saúde, remetendo à intersetorialidade.

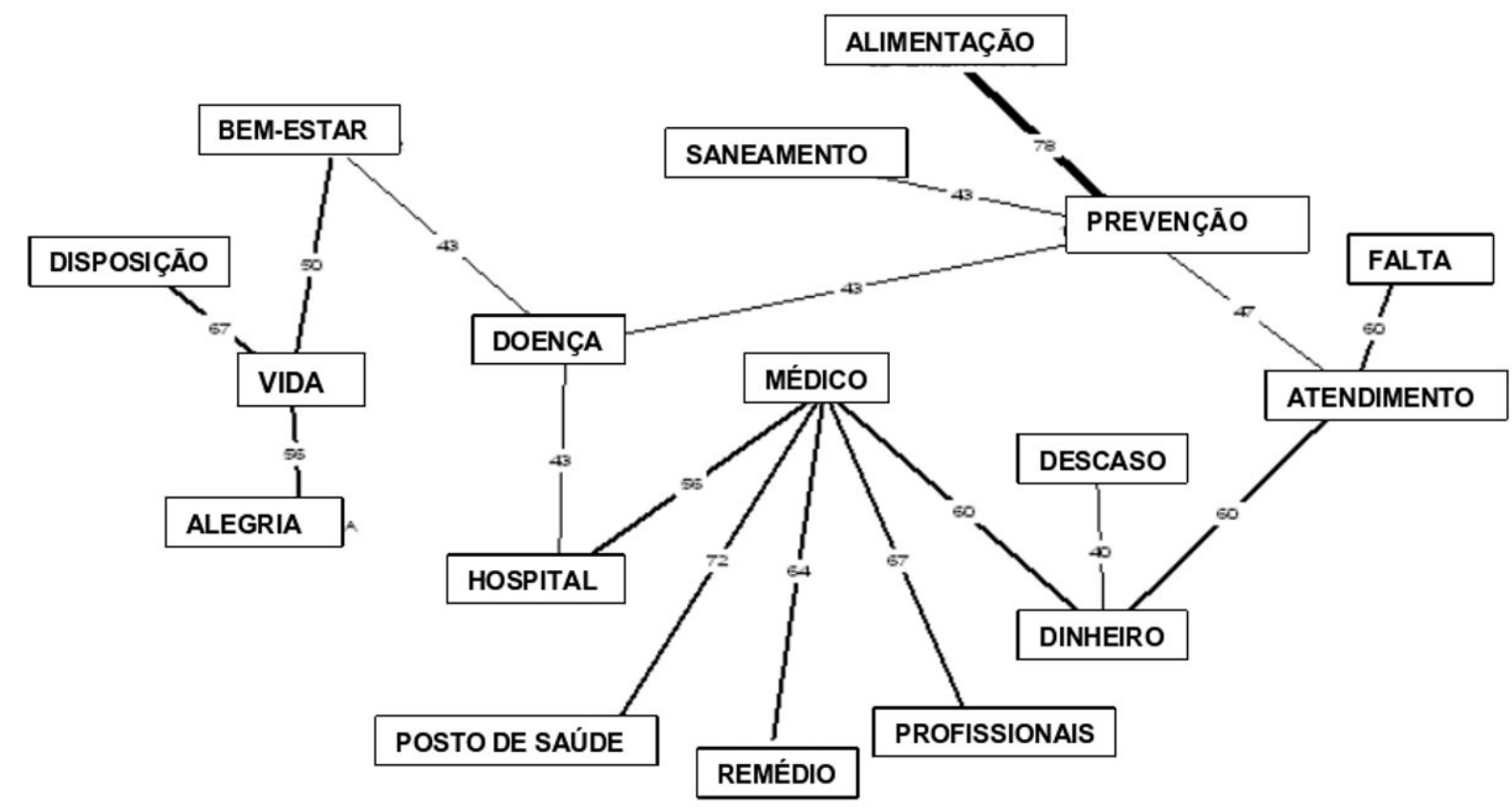

Figura 1 - Árvore máxima de similitude.

\section{CONSIDERAÇÕES FINAIS}

A questão do envelhecimento se configura como um processo de mudança e, como tal, demanda um ritmo contínuo de estudos que busquem contemplar os diferentes aspectos que emergem. $O$ desafio que está posto é a articulação de diferentes campos do conhecimento relacionados ao processo saúde-doença.

Os sentidos atribuídos à saúde pelo grupo que participou dessa investigação constituem um conjunto de informações relevantes, indicando a visão de sujeitos que vivem em contextos socioculturais e econômicos muito diferenciados e em uma região que tem demandado a implantação de políticas especialmente orientadas para suas especificidades locais. $\mathrm{O}$ que se teve oportunidade de ver emergir, nesse caso, foi uma série de dados que indicam as preocupações cotidianas desse grupo de idosos com a falta de infraestrutura assistencial, elencando aspectos qualitativos e quantitativos do problema, mostrando também que haviam apreendido nos processos de comunicação social outros conteúdos mais relacionados com o paradigma da promoção da saúde. Esses conteúdos têm-se apresentado atualmente como uma interface ou o outro lado daquilo que se convencionou distinguir como o atendimento médico. Este contexto adquire maior relevância diante da perspectiva de pavimentação da rodovia federal BR-163, que atravessa a região e que pode - em suas muitas interferências no meio ambiente - trazer incremento dos agravos à saúde.

Os resultados indicam que esse grupo está atento a suas necessidades e tem discutido sobre isso, pois informações mais recentes - como aquelas que se relacionam com a promoção da saúde - já 
fazem parte de seus discursos. Demonstram também que atenção à saúde recebida por esse grupo se caracteriza pela fragmentação, ou seja, reconhecem a importância das atitudes e práticas individuais relacionadas com a promoção da saúde, valorizam o acesso à assistência e medicamentos proporcionados pela expansão do Programa de Saúde da Família, mas se ressentem da inexistência de um sistema integrado que assegure a continuidade do cuidado.

\section{REFERÊNCIAS}

1. Kalache A. Fórum: envelhecimento populacional e as informações de saúde da PNAD, demandas e desafios contemporâneos. Posfácio. Cad. Saúde Pública 2007; 23(10): 2503-05.

2. Ramos LR, et al. Significance and management of disability among urban elderly residents in Brazil. J Cross Cultural Gerontol 1993; 8: 313-23.

3. Chaves M. Complexidade e transdisciplinaridade: uma abordagem multidimensional do Setor Saúde. Rev Bras Educação Médica 1998: 22(1): 7-18.

4. Puska P, Keller I. Primary prevention of noncommunicable diseases: experiences from population based intervention in Finland for the global work of WHO. Z.Kardiol. 2004; 93(Suppl 2): II37-42.

5. Silvestre JAN, Costa Neto MM. Abordagem do idoso em programas de saúde da família. Cad. Saúde Pública 2003; 19(3): 839-47.

6. Assis A. Novos modelos de assistência à saúde do idoso: desafios e tendências da arquitetura frente ao envelhecimento populacional brasileiro. Rio de Janeiro.Dissertação ( Mestrado em Arquitetura) - Universidade Federal do Rio de Janeiro; 2006.

7. Pellegrini Filho A. Pesquisa em saúde, política de saúde e eqüidade na América Latina. Ciênc Saúd Colet 2004; 9(2): 339-50.

8. Moscovici S. Representações Sociais: investigação em psicologia social. Petrópolis: Vozes; 2003.

9. Arruda A. Novos significados da saúde e as representações sociais. Cad Saúd Colet 2002; 10(2):215-27.

10. Jodelet D. Representações sociais: um domínio em expansão. In: Jodelet D . As representações sociais. Rio de Janeiro: EDUERJ; 2001. p. 17-44

11. Rezende TB. Idosos e a consulta médica: um estudo de representações sociais. Rio de Janeiro. Dissertação [Mestrado em Saúde coletiva].Universidade Federal do Rio de Janeiro; 2003.

12. Madeira M. Representações Sociais e Educação: importância teórico-metodológica de uma

relação. In: MOREIRA ASP. Representações Sociais: teoria e prática. 2. ed. João Pessoa: EDUFPB; 2003. p.123-44

13. DATASUS/MS. Ministério da Saúde. Disponível em www.datasus.gov.br Acessado em 09/04/2010.

14. Loyola Filho AI, et al. Causas de internações hospitalares entre idosos brasileiros no âmbito do Sistema Único de Saúde. Epidemiologia e Serviços de Saúde 2004; 13(4): 229 - 38.

15. Abric JC. L'analyse structurale des représentatrions socials. In: Moscovici S, Buschini F. Les méthodes des sciences humaines. Paris: PUF; 2003. p. 375-92.

16. Sá CP. Núcleo central das representações sociais. Petrópolis: Vozes; 1996.

17. Vergès P. A evocação do dinheiro: um método para a definição do núcleo central de uma representação. In. MOREIRA ASP, et al. Perspectivas teórico-metodológicas em representações sociais. João Pessoa: EDUFPB; 2005. p. 471-88.

18. Tura LFR. Aids e estudantes: a estrutura das representações sociais. In: Jodelet D, Madeira MC. Aids e representações sociais: à busca de sentidos. Natal: EDUFRN; 1998. p.121-54.

19. Verges P, Scano S, Junique C. Ensemble de programmes permettant l'analyse des evocations. EVOC2000: manuel. [France?]: LAMES; 2000.

20. Flament C. Aspects périphériques des répresentations sociales. In: Guimelli C. Directeur. Structures et transformations des représentations sociales. Lausanne: Delachaux et Niestlé ; 1994. p. 85-115

21. Bouriche B. L' analyse de similitude. In: Abric J-C. Méthodes d'étude des représentations sociales. Saint-Agne: Éditions Érès ; 2003. p. 221-52.

22. Pereira FJC. Análise de dados qualitativos aplicados às representações sociais. In. Moreira ASP, et al. Perspectivas teórico-metodológicas em representações sociais. João Pessoa: EDUFPB, 2005; p. 25-60. 
23. Bardin L. L'analyse de contenu et de la forme des comunications. In: Moscovici S, Buschini F. Les méthodes des sciences humaines. Paris: PUF, 2003. p. 243-70.

24. Barreto KML, et al. Perfil sócio-epidemiológico demográfico das mulheres idosas da Universidade Aberta à Terceira Idade no estado de Pernambuco. Rev. Bras. Saude Mater. Infant. 2003;3(3):339-54.

25. Lebrão ML, Laurenti R. Saúde, bem-estar e envelhecimento: o estudo SABE no Município de São Paulo. Rev Bras Epidemiol 2005; 8(2): 127-41.

Recebido: 05/1/2011

Revisado: 25/4/2011

Aprovado: 23/8/2011
26. Santos MRDR, et al. Caracterização nutricional de idosos com hipertensão arterial em Teresina. Rev. Bras. Geriatr. Gerontol. 2007; 10(1). [acesso 22 mar. 2010]. Disponível em: http://www.unati.uerj.br

27. Zaitune MPA, et al. Hipertensão arterial em idosos: prevalência, fatores associados e práticas de controle no Município de Campinas. Cad. Saúde Pública 2006; 22(2): 285-94, 2006.

28. Veras R. Envelhecimento populacional contemporâneo: demandas, desafios e inovações. Rev Saúde Pública 2009; 43(3): 548-54.

29. Orvig AS. Éléments de sémiologie discursive. In: Moscovici S, Buschini F. Les méthodes des sciences humaines. Paris: PUF; 2003. p. 271-95. 